\title{
Salvage of infected cardiac implantable electronic device with taurolidine-a case report
}

\author{
Stefan Borov ${ }^{1}$, Benito Baldauf ${ }^{*}$ (D), Ernest W. Lau ${ }^{3}$ and Hendrik Bonnemeier ${ }^{2}$
}

\begin{abstract}
Background: Cardiac implantable electronic devices (CIEDs) are commonly used to treat cardiac arrhythmias and prevent sudden cardiac death. Complications of CIED therapy include component malfunction, lead dislodgement, skin erosion and infection. Infection can result in significant morbidity and even mortality. The recommended treatment of CIED skin erosion and infection is urgent complete device extraction. When this is infeasible due to patient or resource factors, an attempt could be made to salvage the exposed or infected CIED system by debridement of all the infected necrotic tissues and irrigation of the pocket and contaminated hardware with anti-septic/antibiotic solutions. Taurolidine, when dissolved in an aqueous solution, produces a broad spectrum of antimicrobial actions and may be used as a novel irrigation agent during CIED salvage.

Case presentation: This report describes the first use of a taurolidine-containing solution for pocket irrigation and in situ hardware sterilisation that resulted in the successful salvage of a CIED infected with multi-resistant Staphylococcus epidermidis.

Conclusions: A taurolidine-containing antimicrobial solution can be a safe and effective alternative to traditional antiseptic/antibiotic solutions for pocket irrigation and in situ hardware sterilisation during CIED salvage, and may produce better clinical outcomes by some unique mechanisms of action such as inhibition of biofilm formation and neutralisation of endotoxins, with little risk of inducing and encountering resistance.
\end{abstract}

Keywords: Cardiac implantable electronic device, Surgical site infection, Revision, Taurolidine, Case report, Salvage

\section{Background}

Cardiac implantable electronic devices (CIEDs) deliver lifesaving and transforming therapies for millions of patients worldwide every year. Globally, CIED implantation has been increasing year-on-year in many countries [1]. However, the growth in CIED implantation has coincided with a higher-than-expected rise in CIED infection $[2,3]$. The reasons of the excess rise in CIED infection are many and include older CIED recipients with more comorbidities and a larger portion of complex and revision

\footnotetext{
*Correspondence: sportmedic@gmail.com

${ }^{2}$ Medical Faculty, Christian Albrechts Universität, Christian-Albrechts Platz

4, Kiel, Germany

Full list of author information is available at the end of the article
}

procedures (e.g. pulse generator replacement, lead insertion and removal) $[4,5]$. The current guidelines recommend complete removal (including all the leads) of any infected CIED system [3]. However, this radical treatment strategy might not always be feasible due to patient and resource factors, in which case an attempt could be made to salvage the infected CIED system [6]. Successful salvage of infected CIED system with in situ sterilisation of the contaminated hardware has been reported $[7,8]$.

TauroPace $^{\mathrm{TM}}$ (TauroPharm GmbH, Waldbüttelbrunn, Bavaria, Germany) is an antimicrobial agent specifically developed for use during CIED procedures. Its active ingredient is taurolidine. Dissolved in an aqueous solution (which contains povidone to increase its stability), in presence of esterase and enzymes, taurolidine and 
cyclo-taurolidine are hydrolysed to the active compounds n-methylol-taurultam, taurultam and n-methylol-taurinamide $[9,10]$. The metabolites degrade further through hydrolysis into taurine, a naturally occurring aminosulfonic acid found in many biological tissues and secretions. In the process, 3 methylol groups are generated and these are believed to be the chemical active species responsible for taurolidine's antimicrobial actions, which include denaturation of the pathogen cell wall and surface fimbrae or flagellate, inhibition of adherence to human epithelial and fibroblast cells, interference with biofilm formation, neutralisation of endotoxins and some exotoxins [11-15]. After all the antimicrobial actions have been delivered, the end-product of taurolidine metabolism, taurine, has anti-oxidant and anti-inflammatory properties and has been shown to enhance wound healing in vitro and in animal models in vivo [16-18]. Taurine creates an acidic local environment that further enhances the hydrolysis of taurolidine. The half-lives of the active metabolites methyl-taurultam and methylol taurinamide are 1.56 and $6 \mathrm{~h}$ in the presence of saccharides, peptides and glycans [19].

\section{Case presentation}

A 70-year-old male patient was implanted with a single-chamber implantable cardioverter defibrillator (ICD) in February 2012. In February 2017, his device was upgraded to an ICD capable of delivering cardiac resynchronisation therapy. The upgrade procedure was straightforward and the patient received antibiotic prophylaxis as per the implantation centre's protocol. The patient made an uncomplicated recovery from the procedure. He had no issues with device malfunction or wound infection/skin erosion at 4 weeks and 12 weeks follow-up.

In November 2018, the patient was incidentally noticed to have purulent fluid seeping out from a fistula approximately $8 \mathrm{~mm}$ cranial to the lateral end of the device wound scar when assessed for toe amputation (Fig.1). The patient was re-referred back to the electrophysiology service. On inspection, the inflammation appeared mild and localised around the device wound scar. Swabs from the discharging punctum grew methicillin-resistant Staphylococcus epidermidis sensitive to linezolid. Repeated blood cultures were consistently negative for bacterial growth. Trans-oesophageal echocardiography showed no vegetations on the intracardiac portions of the leads. The patient declined complete ICD explantation with lead extraction but would agree to a less invasive device salvage procedure. As a result, the latter treatment strategy was adopted.

Pre-procedure, empirical antibiotic therapy was started with cefuroxim. It was changed and the patient received 7 continuous days of intravenous linezolid as per the

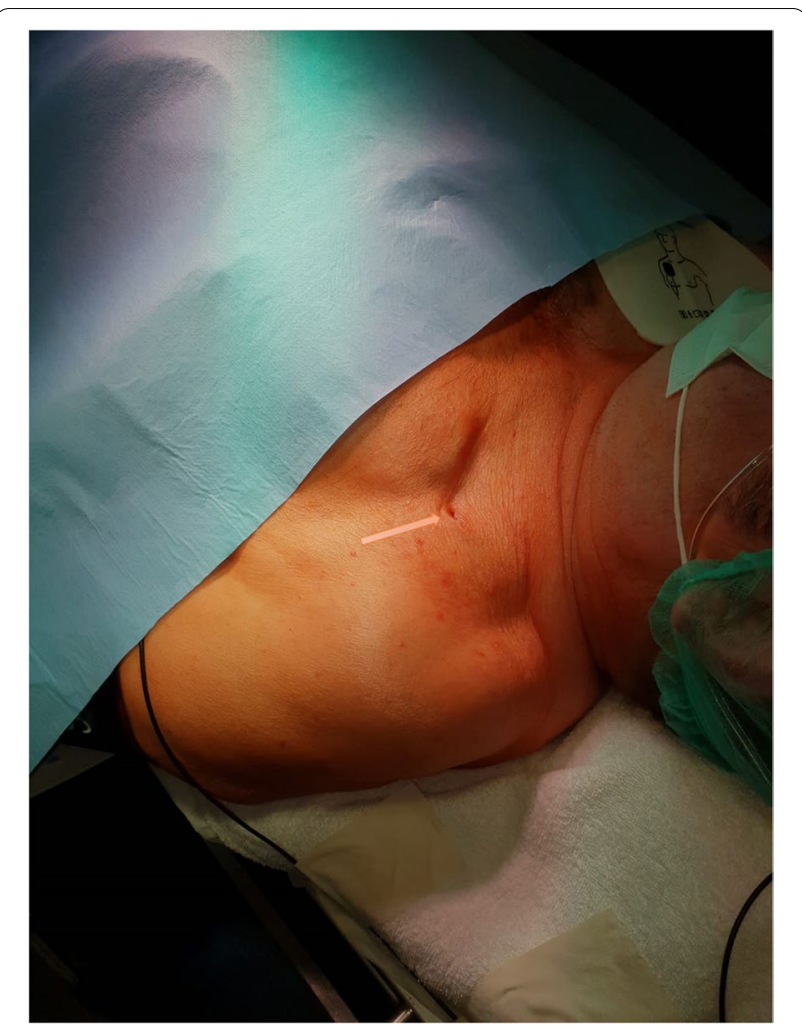

Fig. 1 Picture of surgical site showing discharging punctum just cranial to the lateral end of the previous incision scar

advice from Microbiology. During the procedure, an elliptical island of skin around the punctum was excised. The incision was then extended into the original ICD wound scar at both ends. The ICD pulse generator was liberated from the encasing fibrous subcutaneous tissues, extracted out of the device pocket, detached from the leads, cleaned of any obvious adherent debris and then immersed in TauroPace ${ }^{\mathrm{TM}}$ (TauroPharm GmbH, Bavaria, Germany). The sutures holding the anchor sleeves and leads in place were cut and removed. The anchor sleeves were removed. Capsulectomy was performed by electric cautery with simultaneous haemostasis. The debrided device pocket was irrigated with TauroPace ${ }^{\mathrm{TM}}$. The accessible segments of the leads were wiped with swabs soaked with TauroPace ${ }^{\mathrm{TM}}$. The connector pins of the leads were wiped with TauroPace ${ }^{\mathrm{TM}}$ swabs before being plugged back into the ports on the old ICD pulse generator. The torque wrench was dipped in TauroPace ${ }^{\mathrm{TM}}$ before engaging with the seals covering the set screws. A subpectoral pocket was prepared and irrigated with TauroPace ${ }^{\mathrm{TM}}$ and the pulse generator and leads were positioned within. The wound was closed. Another $20 \mathrm{cc}$ of TauroPace ${ }^{\mathrm{TM}}$ was injected directly into the subcutaneous tissues around the wound (not into the pocket!). 
The device wound showed some mild inflammation the next day but that rapidly settled. Repeat blood culture was negative for bacterial growth. The patient recovered well from the procedure and was discharged. The patient was followed up continuously for 33 months from his device salvage in November 2018 till his death from causes unrelated to his ICD or infection in August 2021 (Fig. 2). During that time, the patient suffered no recurrence of ICD infection or skin erosion.

\section{Conclusions}

Despite categorical guideline recommendation of complete system explantation for treatment of CIED infection [3], lead extraction requires special equipment and expertise, may not be readily available or acceptable to the patient, and is associated with a risk (however small) of very serious complications (e.g. cardiac avulsion, vascular avulsion, haemothorax and cardiac tamponade) that may result in emergency open chest surgery or even death [20]. CIED salvage is an attractive alternative management strategy if it could be made more effective in "eradicating" infection, in the sense of no recurrence of overt clinical infection even if dormant bacteria colonisation remains [21]. CIED salvage may have been successful in anecdotal reports [7, 8], but was not consistently effective in larger series [6]. The antimicrobial agents which have been used for pocket irrigation and in situ hardware sterilisation include diluted povidone-iodine, diluted hydrogen peroxide, diluted alcohol and antibiotic solutions. TauroPace ${ }^{\mathrm{TM}}$ is an alternative antimicrobial agent that has been successfully used to salvage left ventricular assist device driveline infection [22]. Apart from

\section{Primary implant VVIR-ICD 2012}

STEMI 1990 with CABG LIMA-LAD and CABG RCA, Re-CABG 2002

$12 \mathrm{PCl}$ from 2000-2016

Mitra-Clip 2015

PAD - exarticulation digitus 1 right foot 2016

Diabetes mellitus type II, atrial fibrillation, OSAS

\section{Upgrade revision 2/2017 CRT-D}

\section{CIED infection 11/2018} Revision with Taurolidine

No relapse of infection for 33 months - patient succumbed in $8 / 2021$ (complications vascular surgery)

Fig. 2 Timeline 
antimicrobial actions, the wound healing enhancement properties of taurolidine (taurine) when locally applied (directly injected into the subcutaneous tissues around the pocket in this case) is an additional bonus $[17,18]$. This first report of TauroPace ${ }^{\mathrm{TM}} \mathrm{s}$ use in successful salvage CIED infection provides a proof-of-concept and foundation for future research.

\begin{abstract}
Abbreviations
CABG: Coronary artery bypass graft; CIED: Cardiac implantable electronic device; CRT-D: Implantable cardiac synchronisation therapy defibrillator; LAD: Left anterior descending artery; LIMA: Left internal mammary artery; OSAS: Obstructive sleep apnea syndrome; PAD: Peripheral arterial disease; RCA: Right coronary artery; STEMI: ST elevation myocardial infarction; WVIR-ICD: Ventricleventricle-inhibition-rate-modulation implantable cardioverter defibrillator (ICD with one lead/shock coil).
\end{abstract}

\section{Acknowledgements}

Not applicable

\section{Authors' contributions}

S.B. original draft, writing, review and editing; B.B. corresponding author, original draft, writing, review and editing; E.W.L. writing, review and editing; H.B. review and editing, supervision, conceptualisation. The authors read and approved the final manuscript.

\section{Funding}

This research received no funding.

\section{Availability of data and materials}

Please note that no data set was generated due to the nature of this publication. The authors confirm that the data supporting the findings of this study are available in the manuscript.

\section{Declarations}

\section{Ethics approval and consent to participate}

Ethic approval number: 2021 - 1231 from the Bavarian State Medical Chamber Review Board.

\section{Consent for publication}

Obtained in 2018.

\section{Competing interests}

B. Baldauf is a medical consultant for the manufacturer of TauroPace ${ }^{\mathrm{TM}}$. The other authors declare no conflict of interest in relation to this publication.

\section{Author details}

${ }^{1}$ Department of Electrophysiology, Krankenhaus Landshut Achdorf, Landshut, Germany. ${ }^{2}$ Medical Faculty, Christian Albrechts Universität, Christian-Albrechts Platz 4, Kiel, Germany. ${ }^{3}$ Department of Cardiology, Royal Victoria Hospital, Grosvenor Road, Belfast BT12 6BA, UK.

Received: 24 December 2021 Accepted: 4 February 2022

Published online: 16 February 2022

\section{References}

1. Raatikainen, M.J.P., et al., A decade of information on the use of cardiac implantable electronic devices and interventional electrophysiological procedures in the European Society of Cardiology Countries: 2017 report from the European Heart Rhythm Association. Europace, 2017. 19(suppl_2): p. ii1-ii90.

2. Greenspon AJ et al (2011) 16-year trends in the infection burden for pacemakers and implantable cardioverter-defibrillators in the United States 1993 to 2008. J Am Coll Cardiol 58(10):1001-1006
3. Blomström-Lundqvist, C., et al., European Heart Rhythm Association (EHRA) international consensus document on how to prevent, diagnose, and treat cardiac implantable electronic device infections-endorsed by the Heart Rhythm Society (HRS), the Asia Pacific Heart Rhythm Society (APHRS), the Latin American Heart Rhythm Society (LAHRS), International Society for Cardiovascular Infectious Diseases (ISCVID) and the European Society of Clinical Microbiology and Infectious Diseases (ESCMID) in collaboration with the European Association for Cardio-Thoracic Surgery (EACTS). Europace, 2020. 22(4): p. 515-549.

4. Poole JE et al (2010) Complication rates associated with pacemaker or implantable cardioverter-defibrillator generator replacements and upgrade procedures: results from the REPLACE registry. Circulation 122(16):1553-1561

5. Dai M et al (2019) Trends of Cardiovascular Implantable Electronic Device Infection in 3 Decades: A Population-Based Study. JACC Clin Electrophysiol 5(9):1071-1080

6. Peacock JE Jr et al (2018) Attempted salvage of infected cardiovascular implantable electronic devices: Are there clinical factors that predict success? Pacing Clin Electrophysiol 41(5):524-531

7. Schaller RD, Cooper JM (2017) Salvage of focally infected implantable cardioverter-defibrillator system by in situ hardware sterilization. HeartRhythm Case Rep 3(9):431-435

8. Roshdy H, Seaoud E, Elbelbesy R (2021) Low-budget, single-session elimination of CIED pocket infection. Pacing Clin Electrophysiol 44(1):129-134

9. Gong L et al (2007) The pharmacokinetics of taurolidine metabolites in healthy volunteers. J Clin Pharmacol 47(6):697-703

10. Stendel $R$ et al (2007) Pharmacokinetics of taurolidine following repeated intravenous infusions measured by HPLC-ESI-MS/MS of the derivatives taurultame and taurinamide in glioblastoma patients. Clin Pharmacokinet 46(6):513-524

11. William R et al (1995) Taurolidine, an antilipopolysaccharide agent, has immunoregulatory properties that are mediated by the amino acid taurine. Jeukocyte Biol 58(3):299-306

12. Monson JR, Ramsey PS, Donohue JH (1993) Taurolidine inhibits tumour necrosis factor (TNF) toxicity--new evidence of TNF and endotoxin synergy. Eur J Surg Oncol 19(3):226-231

13. Caruso, F., et al., Taurolidine antiadhesive properties on interaction with E. coli; its transformation in biological environment and interaction with bacteria cell wall. PLoS One, 2010. 5(1): p. e8927.

14. BLENKHARN JI (1988) Sustained anti-adherence activity of taurolidine (Taurolin) and noxythiolin (Noxyflex S) solutions. J Pharmacy Pharmacol 40(7):509-511

15. GORMAN SP et al (1987) Electron and light microscopic observations of bacterial cell surface effects due to taurolidine treatment. Letters Appl Microbiology 4(5):103-109

16. Ashkani-Esfahani S et al (2014) Taurine improves the wound healing process in cutaneous leishmaniasis in mice model, based on stereological parameters. Adv Biomed Res 3:204

17. Dinçer S et al (1996) Effect of taurine on wound healing. Amino Acids 10(1):59-71

18. Değim Z et al (2002) An investigation on skin wound healing in mice with a taurine-chitosan gel formulation. Amino Acids 22(2):187-198

19. Brückner, W.L., Taurolin: e. neues Konzept zur antimikrobiellen Chemotherapie chirurg. Infektionen; dieser Bd. enth. d. anlässl. d. Internat. Taurolin-Symposiums am 22. Oktober 1983 in München gehaltenen Vorträge in überarb. u. erw. Form. 1985: Urban \& Schwarzenberg.

20. Kusumoto FM et al (2017) 2017 HRS expert consensus statement on cardiovascular implantable electronic device lead management and extraction. Heart Rhythm 14(12):e503-e551

21. Mason PK et al (2011) Sonication of explanted cardiac rhythm management devices for the diagnosis of pocket infections and asymptomatic bacterial colonization. Pacing Clin Electrophysiol 34(2):143-149

22. Weichsel J et al (2022) Eradication of Ventricular Assist Device Driveline Infection in Paediatric Patients with Taurolidine. J Cardiovasc Dev Dis 9(1):18

\section{Publisher's Note}

Springer Nature remains neutral with regard to jurisdictional claims in published maps and institutional affiliations. 